\title{
Evaluation of supplementary diagnostic value of contrast- enhanced ultrasound for lymph node puncture biopsy
}

\author{
Jie Zhang ${ }^{1 *}$, Xin $\mathrm{Hao}^{2 *}$, Yang Yang ${ }^{3}$, Chang-Shuai Yan ${ }^{2}$, Chao $\mathrm{Ma}^{1}, \mathrm{Ming} \mathrm{Xiao}^{1}$, Li-Shuang Gu${ }^{4}$, Yong Wang ${ }^{4}$ \\ ${ }^{1}$ Department of General Surgery, General Hospital of Tianjin Medical University, Tianjin 300051, China; ${ }^{2}$ Graduate School of Tianjin Medical \\ University, Tianjin 300070, China; ${ }^{3}$ Department of Radiology, Tianjin Children's Hospital, Tianjin 300134, China; ${ }^{4}$ Department of Ultrasound, \\ National Cancer Center/Cancer Hospital, Chinese Academy of Medical Sciences and Peking Union Medical College, Beijing 100021, China \\ Contributions: (I) Conception and design: J Zhang, X Hao, Y Wang, Y Yang; (II) Administrative support: J Zhang, C Ma, Y Wang, Y Yang; (III) \\ Provision of study materials or patients: J Zhang, X Hao, CS Yan; (IV) Collection and assembly of data: J Zhang, X Hao; (V) Data analysis and \\ interpretation: J Zhang, X Hao, C Ma, M Xiao, LS Gu, Y Yang; (VI) Manuscript writing: All authors; (VII) Final approval of manuscript: All authors. \\ *These authors contributed equally to this work. \\ Correspondence to: Yong Wang. Department of Ultrasound, National Cancer Center/Cancer Hospital, Chinese Academy of Medical Sciences \\ and Peking Union Medical College, Panjiayuannanli No. 17, Chaoyang District, Beijing 100021, China. Email: drwangyong77@163.com; \\ Yang Yang. Department of Radiology, Tianjin Children's Hospital, Long Yan Road No. 238, Beichen District, Tianjin 300134, China. \\ Email: houwenjing1986@126.com.
}

Background: Contrast enhanced ultrasound (CEUS) is an effective way to evaluate the distribution of micro vessels in organs with little invasiveness. The qualitative evaluation and differential diagnosis of lymph node is essential for a number of clinical situations, including appropriate examination selection, oncologic staging, therapy and prognosis beyond diagnosis itself. The purpose of this study is to investigate the contribution of characterization of cervical lymphadenopathies on CEUS as an indication for lymph node puncture biopsy.

Methods: Records of 157 lymph nodes from 103 patients who had undergone CEUS examination were studied retrospectively, and enhancement patterns and characteristics were evaluated. The diagnoses obtained by means of core-needle biopsy examination or surgical removal, or patients were closely followed-up for at least 6 months. Of the lymph nodes examined, 94 were benign (48 lymphadenitis and 46 tuberculosis) and 63 were malignant (55 metastases and 8 lymphomas). The vascular characterization of lymph nodes was compared with results obtained by pathologic examination.

Results: The study using CEUS showed 55 (54.5\%) cases of "peripheral" enhancement in group A and $47(83.9 \%)$ cases of "hilar normal or activated" in group B. Inhomogeneous "spotted or cycle-like" enhancement in the equilibrium phase was seen in 80 (79.2\%) cases of group A and only 11 (19.6\%) cases of group B. The specificity, sensitivity and accuracy of CEUS examination result as an indication for puncture biopsy were $80.4 \%, 81.2 \%$ and $80.9 \%$, respectively.

Conclusions: Centripetal perfusion in the early arterial phase or inhomogeneous spotted or cycle-like enhancement in the equilibrium phase of the cervical lymph nodes during CEUS can be used as an indication for core-needle biopsy with a high degree of accuracy. Conversely, for lymph nodes with more variable appearances, surgical removal or closely followed-up of nodes may be more appropriate.

Keywords: Contrast-enhanced ultrasound; cervical lymph node; indication of puncture

Submitted Oct 11, 2017. Accepted for publication Nov 14, 2017.

doi: $10.21037 /$ jtd.2017.11.90

View this article at: http://dx.doi.org/10.21037/jtd.2017.11.90 


\section{Introduction}

Conventional color Doppler sonography is generally considered the method of choice for evaluation of vascularity in enlarged superficial lymph nodes. However, in malignant as well as benign enlarged lymph nodes, the Doppler signal is limited because of the low flow velocity of blood, especially in cases where only some parts of the lymph node were infiltrated by tumour (1). Contrast enhanced ultrasound (CEUS), a modern imaging method for investigation of tissue microvascular perfusion in real time, was demonstrated to be feasible in the differentiation of benign from malignant lymph nodes (2). The qualitative evaluation and differential diagnosis of lymph nodes are fundamental in the diagnosis because they influence not only the assessment of the oncologic stage and prognosis, but also the selection of appropriate invasive tests and treatment monitoring strategies (3). For example, the diagnosis of lymphoma requires histologic examination after surgical removal of the node, whereas cytologic examination after core-needle puncture biopsy is generally sufficient for the diagnosis of metastatic and tuberculous nodes. Different enhancement characteristics in CEUS provide a possibility to choose appropriate lymph node candidate for core-needle puncture biopsy. If CEUS is done early, it would obviate unnecessary core-needle aspiration examination and would lead to completely different diagnostic routes for the patients with suspected lymphomas or lymphadenitis (node excision or follow-up only). To our knowledge, no report has been specifically dedicated to the study of metastatic and tuberculous nodes, which are pathologically different from lymphomatous nodes, to justify a separate evaluation for each. Discussion continues, however, about whether differentiation of their vascular architecture on CEUS is credible and whether CEUS is helpful as a tool for screening out candidates for coreneedle puncture examination. We performed a retrospective study to document the lymph node enhancement patterns and characteristics in CEUS, and we identified special signs that could help in differentiating the two categories before invasive examination.

\section{Methods}

\section{Patient selection}

One hundred and three patients [ 47 men and 56 women, ranging in age from 16 to 80 years (mean, 51.6 years)] with enlarged lymph nodes were recruited, and a total of 157 nodes were found on sonography. The lymph node was considered to be enlarged if its maximal transverse diameter of nodes greater than $5 \mathrm{~mm} \mathrm{(3).} \mathrm{The} \mathrm{diagnosis} \mathrm{obtained} \mathrm{by}$ means of core-needle biopsy, surgical removal, or both, as well as a close follow-up for 6 months at least. Fortyeight of 157 enlarged lymph nodes histologically showed lymphadenitis; 46 were tuberculous; 55 were metastatic; and 8 were lymphomas. The lymph nodes were divided into two groups if a qualitative histopathological result could be acquired from core-needle biopsy only: group A for if the nodes could be differentiated (tuberculosis and metastases) and group B for if the nodes could not be differentiated (lymphadenitis and lymphoma).

During the B-scan sonographic examination, we measured the longitudinal and axial diameters of the lymph nodes. We evaluated also the long/short axis (L/S) ratio, internal echogenicity (whether the parenchyma was homogeneously hypoechoic or contained inhomogeneous or anechoic areas), visibility of the hilum (if it was central and normally shaped, eccentric, irregular, or completely invisible), and Doppler signal (hilar or peripheral vessel) for each node. The L/S ratio less than 2, presence of hypoechoic region without echogenic hilum, and presence of blood vessels predominance in the periphery were considered indicative of malignancy. The lymph nodes were classified as abnormal if at least 2 signs among those considered under conventional ultrasound were present (4).

\section{Imaging technique and interpretation}

We used a MyLab Class C (Esaote, Genoa, Italy) machine equipped with L522 (3-8 MHz) linear-array transducer for CEUS. A 7.5-MHz dedicated linear transducer was used for CEUS examination. The low values of mechanical index and acoustic intensity would preserve the microbubbles from being destroyed. All examinations were performed by the same investigator with 15 years of experience in small glands ultrasound examination. Informed consent was obtained before their examination. The local ethics committee and instructional review board approved this study. A total of $2.5 \mathrm{~g}$ contrast agent (SonoVue: sulfur hexafluoride with a phospholipid shells; Bracco Imaging SpA, Milan, Italy) in a volume of approximately $5 \mathrm{~mL}$ solution was administrated intravenously as a single bolus, and it was followed by flushing of $10 \mathrm{~mL}$ of physiologic saline solution. A maximum dose of $4 \mathrm{~g}$ with a maximum volume of approximately $10 \mathrm{~mL}$ of contrast agent was given in the form of two or three additional smaller boluses for 
Table 1 Lymph nodes enhanced characteristics on CEUS

\begin{tabular}{|c|c|c|c|c|c|}
\hline CEUS & Metastases [55] & Tuberculosis [46] & Lymphadenitis [48] & Lymphoma [8] & Total [157] \\
\hline \multicolumn{6}{|l|}{ In the early arterial phase } \\
\hline Hilar normal or activated & $37(67.3 \%)$ & $9(19.6 \%)$ & $40(83.3 \%)$ & 7 (87.5\%) & $93(59.2 \%)$ \\
\hline \multicolumn{6}{|l|}{ In the equilibrium phase } \\
\hline $\begin{array}{l}\text { Type I: inhomogeneous Spotted or cycle-like } \\
\text { enhancement }\end{array}$ & $37(67.3 \%)$ & $43(93.5 \%)$ & $10(20.9 \%)$ & $1(12.5 \%)$ & $91(58.0 \%)$ \\
\hline
\end{tabular}

CEUS, contrast enhanced ultrasound.

patients with multiple enlarged nodes, if necessary. All CEUS examinations were digitally recorded. The judgment was retrospectively made by an observer blinded to the histologic results. Subsequently, the CEUS findings were compared with histologic findings.

All nodal perfusion and enhancement patterns were digitally recorded. The nodal perfusion and the enhancement patterns were evaluated in the arterial phase and equilibrium phase. During the CEUS examination, we classified the enhancement patterns into 2 categories based on the enhancement location in the early arterial phase: "hilar normal or activated" (a single vascular pole around the hilum with centrifugal presence of small branches or wider and longer ones than the normal) and "peripheral" (the peripheral vessels arranged in the cortex with multiple irregular and tortuous centripetal branches). According to the contrast distribution in the lymph node in the equilibrium phase, we classified the enhancement patterns into the following types: type I, inhomogeneous spotted type (scattered spots or segments of vessels signals distributed in a random order within the node) or cyclelike type (well-defined defects of hilar perfusion); type II, homogeneous enhancement and no enhancement at all in equilibrium phase.

\section{Statistical analysis}

Sensitivity, specificity, and accuracy were evaluated for CEUS with pathologic results served as the gold standard. Statistical analysis included a 2 -tailed $\chi^{2}$ test for categorical parametric data. A Receiver Operating Characteristic (ROC) curve was drawn to assess the diagnostic value of different enhancement patterns in choosing appropriate patients for core-needle puncture biopsy by calculating the area under the curve. Statistical analyses were performed with SPSS version 19.0 software (IBM Corporation, Armonk, NY,
USA), and a $\mathrm{P}<0.05$ was considered statistically significant.

\section{Results}

With CEUS, initial enhancement could be detected approximately $11 \mathrm{sec}$ after completion of the bolus administration, with a subsequent effective scanning window of approximately $5 \mathrm{~min}$.

CEUS enhancement and perfusion patterns are shown in Table 1. The perfusion as determined by CEUS was identified as "hilum normal or activated enhancement" in 93 lymph nodes; "peripheral enhancement" in 64 lymph nodes; "inhomogeneous spotted enhancement" in 61 lymph nodes; and "cycle-like enhancement" in 30 lymph nodes. Scarce or intranodal enhancement defect was seen in 2 metastatic lymph nodes and in 27 tuberculous lymph nodes.

To determine if the three CEUS groups were correlated with the obtained histopathological result, the special signs were combined. Characteristics used for analysis were statistically significant in comparison seen in Figure 1. Considering the presence of peripheral perfusion as an indication for core-needle biopsy, we obtained 55 lymph nodes as true positive, 47 as true negative, 9 as false positive, and 46 as false negative results. Specificity, sensitivity, and accuracy were $83.9 \%(47 / 56), 54.5 \%(55 / 101)$, and $65.0 \%$ $(102 / 157)$, respectively $\left(\chi^{2}=21.98, \mathrm{P}<0.001\right)$.

Considering the presence of inhomogeneous spotted or cycle-like enhancement as an indication for puncture, we obtained 125 correct diagnoses in nodes whose biopsy results had a clear diagnosis. Specificity, sensitivity, and accuracy were $80.4 \%$ (45/56), 79.2\% (80/101), and 79.6\% (125/157), respectively. $\left(\chi^{2}=52.46, \mathrm{P}<0.001\right)$

In particular, analysis of the CEUS results showed that when presence of "peripheral perfusion" or "spotted or cycle-like enhancement" was used as an indication for coreneedle biopsy, the sensitivity, specificity and accuracy level 


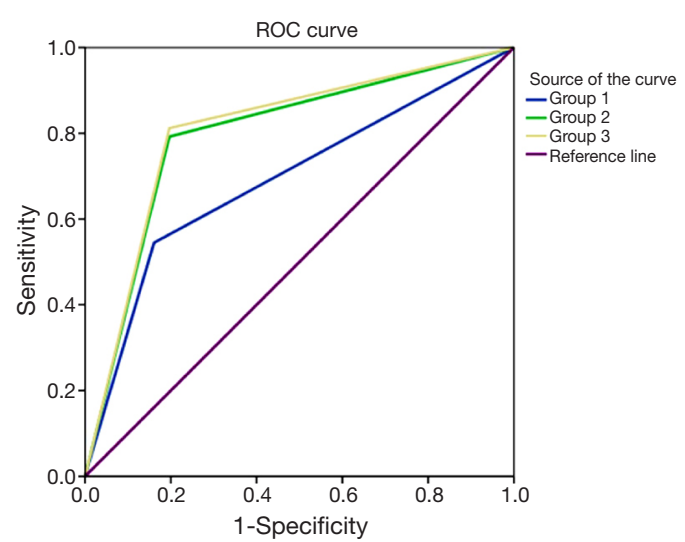

Area under the ROC curve:

\begin{tabular}{|l|c|c|c|c|c|}
\hline Test result & \multirow{2}{*}{$\begin{array}{c}\text { Area } \\
\text { variable(s) }\end{array}$} & Std. & Asymptotic & Asymptotic 95\% confidence interval \\
\cline { 5 - 6 } & & error & sig. & Lower bound & Upper bound \\
\hline Group1 & 0.692 & 0.043 & 0.000 & 0.608 & 0.776 \\
\hline Group2 & 0.798 & 0.039 & 0.000 & 0.722 & 0.874 \\
\hline Group3 & 0.808 & 0.038 & 0.000 & 0.733 & 0.882 \\
\hline
\end{tabular}

Figure 1 ROC curve to assess the diagnostic value of three groups of enhancement patterns in choosing appropriate patients for coreneedle puncture by calculating the area under the curve (Group 1: peripheral perfusion in the arterial phase; Group 2: inhomogeneous spotted or cycle-like enhancement in the equilibrium phase; Group 3: peripheral perfusion in the arterial phase or inhomogeneous spotted or cycle-like enhancement in the equilibrium phase). ROC, Receiver Operating Characteristic.

were $80.4 \%$ (45/56), 81.2\% (82/101) and 80.9\% (127/157), respectively $\left(\chi^{2}=56.51, \mathrm{P}<0.001\right)$, being superior to the two groups above.

The study showed 55 (54.5\%) cases of "peripheral" enhancement in group A and 47 (83.9\%) cases of "hilar normal or activated" in group B. Inhomogeneous "spotted or cycle-like" enhancement in the equilibrium phase was seen in $80(79.2 \%)$ cases of group A and only 11 (19.6\%) cases of group B. The majority of lymph nodes with tuberculosis $(80.4 \%)$ showed vessels arranged in the periphery of the node, while hilum like vessels could be additionally detected in only $9(19.6 \%)$ cases. However, 40 (87.5\%) lymph nodes with lymphadenitis showed hilar vessels branching in the center, which is similar to that in lymphoma with wider and tortuous vessels only (Figure 2).

Eleven lymph nodes had false-positive finding on CEUS scanning. On the basis of histologic examination, the diagnosis was lymphoma in one, necrotic lymphadenitis in seven, and reactive lymph nodes in three: all of these showed areas of intranodal perfusion defect (Figure 3).

\section{Discussion}

CEUS is a promising technique for the investigation of microvascular details of lymph nodes. It can provide specific information about vascular enhancement patterns and defects without an appreciable increase in examination duration and invasiveness (5). Tumor angiogenesis alters the vascular architecture of malignant lymph nodes, and this could give important diagnostic hints (3). Recent literature has indicated the possibility of differentiating malignant nodes from benign ones by observing the enhanced patterns on CEUS. All authors agree that "hilar normal", i.e., a single vascular pole around hilum with linear and regular branches, is a sign of benignity; in contrast, multiple peripheral poles with distortion and displacement of the internal vessels-these being responsible for the "inhomogeneous spotted or cycle-like enhancement"generally indicate malignancy $(2,6)$. Clinically, most diagnoses of lymphomas require histologic examination after surgical removal of the nodes, whereas cytologic examination after core-needle puncture biopsy is generally sufficient for the diagnosis of metastatic and tuberculous nodes. A specific, easy-to-operate technique for screening nodes for which histopathologic results could be achievable by core-needle biopsy could obviate unnecessary examinations and shorten the diagnosis cycle, which would be beneficial for patients with suspected malignant lymph nodes.

As shown in our study by using conventional ultrasound and CEUS, most metastatic and tuberculous lymph nodes have specific vascular perfusion characteristics based on their vascular architecture. These characteristics are different from those of inflammatory and lymphomatous nodes, which mean that this method could be considered as an excellent technique for screening appropriate patients for core-needle puncture.

Lymph node metastases at the time of diagnosis of a tumor have a very significant impact on both the type of treatment chosen and prediction of tumor recurrence. However, preoperative imaging examinations, including US, CT and MRI, have low sensitivity (20-40\%) for the detection and diagnosis of lymph node metastases (7). In metastasis, neoplastic cells travel through afferent lymphatic vessels from the surrounding node, and then colonize the marginal sinus where the disease eventually infiltrates from the periphery to the center (5). Further, neoplastic tumor cells can secrete various kinds of angiogenic factors that simulate growth and formation of de novo vessels. These vessels differ from preexisting vessels and produce abnormal hemodynamics features, such as arterial-venous shunts 

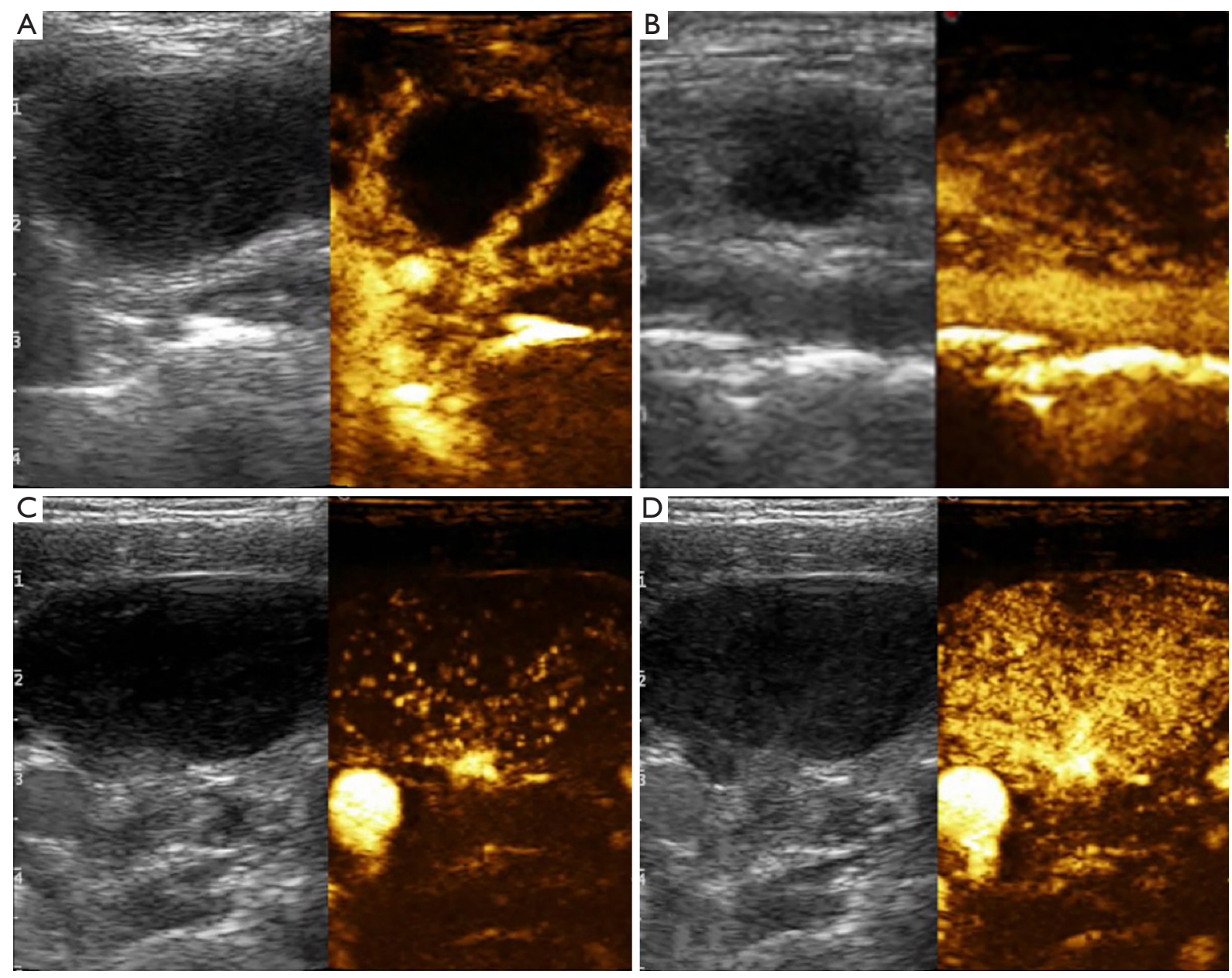

Figure 2 Characteristic enhancement patterns for kinds of disease confirmed by pathologic examination. (A) Tuberculosis: female, 28 years old, CEUS shows a cycle-like enhancement in the equilibrium phase; (B) metastases: female, 57 years old, CEUS shows inhomogeneous spotted enhancement in the equilibrium phase; (C,D) lymphoma: male, 33 years old, CEUS shows progressive centrifugal enhancement pattern like a "snowstorm" in the arterial phase, diffuse and uniform homogeneous enhancement in the parenchymal phase. CEUS, contrast enhanced ultrasound.

due to lack of a muscular layer (8). In addition, neoplastic tissue may compress and even encase a vessel, increasing pressure in the parenchyma and decreasing the blood flow. This may explain the inhomogeneous enhancement and less vascularized lymph nodes in most metastases as compared to the healthy lymph nodes. In our study, all metastases had "inhomogeneous spotted or cycle-like enhancement" in the equilibrium phase, the vessels were typically observed first in the cortical area of the nodes in the arterial phase on CEUS. The enhanced mode shows some relationship with the histologic type. However, for early metastases, which are too minute to alter the vascular architecture and actual morphologic characteristics of the lymph node, the peripheral perfusion can be difficult to detect (5).
Tubercular lymph nodes have a variety of vascular enhancement patterns. Apparent avascularity and aberrant vascularity signal with centripetal flow is also commonly seen. CEUS can also discriminate small necrotic areas in lymph node, which are commonly seen in tuberculous lymphadenopathy. About $80.4 \%$ of tuberculous lymph nodes in our study were generally demonstrated to have peripheral perfusion with thick enhanced septa inside; the result is similar to that described by Yang et al. (9). Lymph node metastases are also generally less vascularized, and therefore are frequently observed as areas of perfusion defects (10). However, necrosis is not specifically found in metastatic and tubercular lymph nodes; although rarely, it can also be observed in necrotic lymphadenitis, which is an 

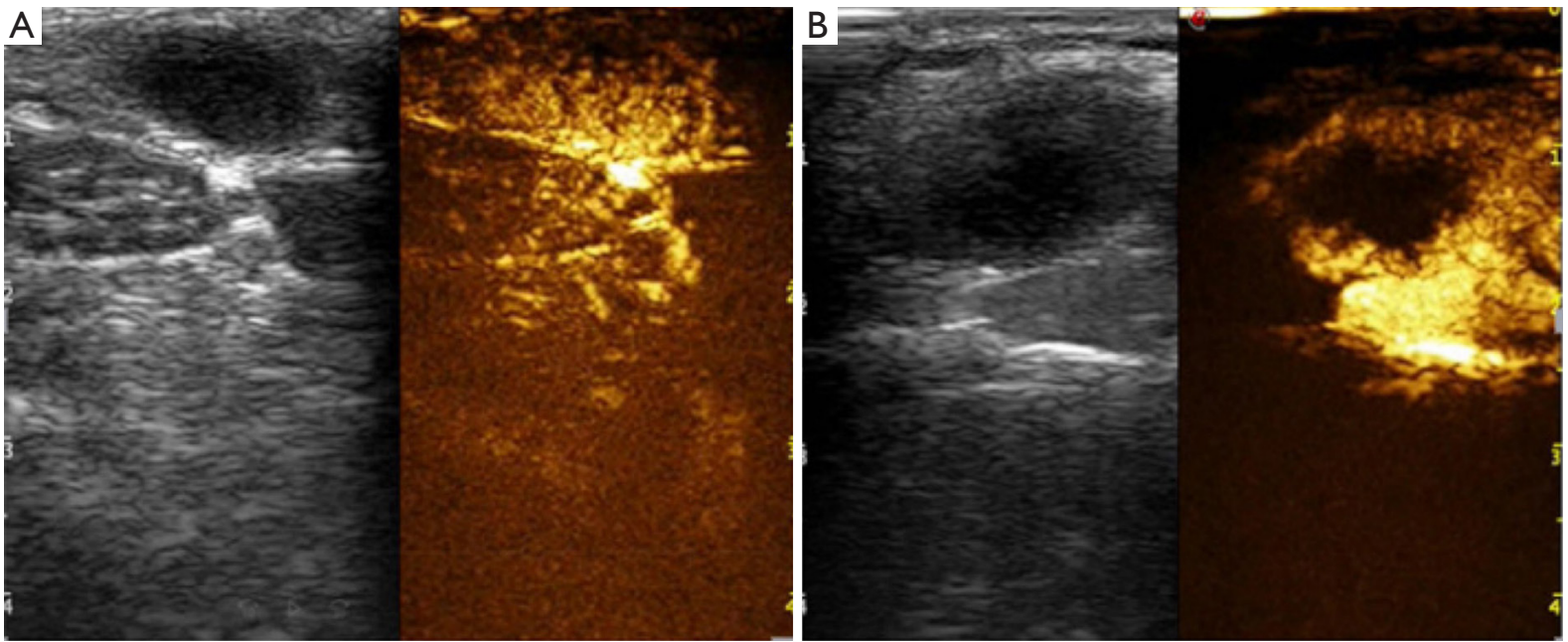

Figure 3 Above shows comparison of two different enhancement patterns in lymphadenitis. (A) Reactive lymph node: female, 32 years old, CEUS shows homogeneous enhancement in the equilibrium phase; (B) necrotic lymphadenitis: female, 57 years old. This is a false positive case. CEUS shows that an enhancement defect in center was misinterpreted as a "cycle-like enhancement", and contradicted by pathologic test. CEUS, contrast enhanced ultrasound.

inflammation process (11). This may explain the finding in the 10 cases of benign lymph nodes with perfusion defects in this study, which were considered to be false-positive for puncture. The morphologic characteristics were highly altered in each of these cases. In addition, hilar fat as an indicator of benign disease can be misinterpreted as central necrosis (12). Mechanism for perfusion defects needs to be further and deeply researched.

Lymphomas are a common cause of superficial cervical lymph node enlargement. In lymphoma, the pathological changes frequently begin inside the lymph node, and may never reach the subcapsular area, depending on the aggressiveness and natural history. Lymphomatous infiltration of the lymph nodes does not produce corresponding changes in the architecture (13), so the affected lymph nodes have preserved hilum normal or activated vessel. Development of the new cortical capillary circulation from the main artery and its branches produces vessel enlargement and intense vascularization. This progression accounts for a homogenous centrifugal fashion in CEUS. Thus, it is difficult to differentiate between lymphadenitis and lymphoma because of rich vascularization distributed in a regular manner (14). Eight lymph nodes were affected by lymphoma in our study, seven cases showed diffused "snowstorm" shaped enhancement in the early arterial phase, with subsequent widespread homogeneous enhancement in the whole node. This is similar to the enhancement perfusion described by Rubaltelli in lymphoma (4). To our surprise, this enhancement pattern was only encountered in the vasculature in lymphomas when compared to other abnormal arterioles. However, the lymph node, as with metastasis, can also be transfected and infiltrated by surrounding preexisting lymphomatous nodes (5). In our study, only one case of lymphoma showed peripheral vascularity: pathologic examination revealed that it was affected by high-grade aggressive Hodgkin lymphoma. The neoplastic cells originated from outside in another lymph node and subsequently infiltrated the examined node.

Reactive lymph nodes characteristically show hilar vessels branching in the center of nodes, which represent the natural entrance for blood vessels entering the node (5). This explains the appearance of the normal or activated homogenous enhancement that we observed in $83.3 \%$ (40/48) of reactive lymph nodes. Histologically, these nodes show an intact nodal architecture with blood vessels located along the hilum and enlarged sinuses due to sinusoidal histiocytosis $(5,15)$.

In summary, the periphery of the node is infiltrated immediately in most metastases, whereas, in lymphoma, the pathologic changes often begin inside the node, it is the center of the node that will be immediately infiltrated, and the periphery may remain normal for a long time. This histologic difference explains the peripheral neovascularity observed in metastasis and tuberculosis and the "hilar normal or activated" vascularity in most lymphoma and lymphadenitis $(6,15)$, at least initially. Without vascular deformation, acutely 
inflamed lymph nodes are difficult to distinguish from lymphoma on basis of enhanced pattern alone (3).

These data imply that CEUS, as a redefined in nodal evaluation, should be taken into account when carrying out core-needle biopsy. Only lymph nodes with suspicious characteristics ("peripheral perfusion" or "spotted or cyclelike enhancement") on CEUS should be considered the most suitable candidate for puncture with a high positive rate. Conversely, for lymphoma with more variable appearance and complicated clinical types, surgical removal of nodes should be first priority.

The usage of core-needle biopsy guided by image technique for suspicious nodes could be beneficial for diagnosis, only when the lymph nodes have specific imaging indication. As a noninvasive CEUS examination can provide credible indication for puncture, it may be considered a supplementary sensitive examination choice for the purpose of screening appropriate candidates.

\section{Acknowledgements}

Funding: This work was supported by Beijing Hope Run Special Fund of China Cancer Research Foundation (CCRF) (No. LC2016A04).

\section{Footnote}

Conflicts of Interest: The authors have no conflicts of interest to declare.

Ethical Statement: The local ethics committee and instructional review board approved this study.

\section{References}

1. Chang DB, Yuan A, Yu CJ, et al. Differentiation of benign and malignant cervical lymph nodes with color Doppler sonography. AJR Am J Roentgenol 1994;162:965-8.

2. de Stefano G, Scognamiglio U, Di Martino F, et al. The role of CEUS in characterization of superficial lymph nodes: a single center prospective study. Oncotarget 2016;7:52416-22.

3. Moritz JD, Ludwig A, Oestmann JW. Contrast-enhanced color Doppler sonography for evaluation of enlarged cervical lymph nodes in head and neck tumors. AJR Am J Roentgenol 2000;174:1279-84.

4. Rubaltelli L, Khadivi Y, Tregnaghi A, et al. Evaluation of lymph node perfusion using continuous mode harmonic ultrasonography with a second-generation contrast agent. J Ultrasound Med 2004;23:829-36.

5. Giovagnorio F, Galluzzo M, Andreoli C, et al. Color Doppler sonography in the evaluation of superficial lymphomatous lymph nodes. J Ultrasound Med 2002;21:403-8.

6. Kong J, Zhang B, Li JC. Contrast-enhanced ultrasonography in differentiation of malignant from benign cervical lymph nodes. Chin J Med Ultrasound (Electronic Edition) 2015;12:108-11.

7. Sumi M, Ohki M, Nakamura T. Comparison of sonography and CT for differentiating benign from malignant cervical lymph nodes in patients with squamous cell carcinoma of the head and neck. AJR Am J Roentgenol 2001;176:1019-24.

8. Steinkamp HJ, Wissgott C, Rademaker J, et al. Current status of power Doppler and color Doppler sonography in the differential diagnosis of lymph node lesions. Eur Radiol 2002;12:1785-93.

9. Yang GY, Zhao D, Huang JY, et al. Contrast enhanced ultra-sonographic features of cervical tuberculous lymphadenitis. Chin J Clin Infect Dis 2010;3:277-9.

10. Galiè $M, D^{\prime}$ Onofrio $M$, Montani $M$, et al. Tumor vessel compression hinders perfusion of ultrasonographic contrast agents. Neoplasia 2005;7:528-36.

11. Kodet R, Campr V, Kalinová M, et al. Histiocytic necrotizing lymphadenitis/Kikuchi-Fujimoto disease (HNL/K-F) and its differential diagnosis: analysis of 19 patients. Cesk Patol 2012;48:198-206.

12. van den Brekel MW, Stel HV, Castelijns JA, et al. Cervical lymph node metastasis: assessment of radiologic criteria. Radiology 1990;177:379-84.

13. DePeña CA, Van Tassel P, Lee YY. Lymphoma of the head and neck. Radiol Clin North Am 1990;28:723-43.

14. Zenk J, Bozzato A, Hornung J, et al. Neck lymph nodes: prediction by computer-assisted contrast medium analysis? Ultrasound Med Biol 2007;33:246-53.

15. Zhang R, Wang XR, Song T, et al. Clinical application value of contrast-enhanced ultrasonography in diagnosis of cervical lymphadenopathy. Chinese J Ultrasound Med 2016;32:392-5.

Cite this article as: Zhang J, Hao X, Yang Y, Yan CS, Ma C, Xiao M, Gu LS, Wang Y. Evaluation of supplementary diagnostic value of contrast-enhanced ultrasound for lymph node puncture biopsy. J Thorac Dis 2017;9(11):4791-4797. doi: 10.21037/jtd.2017.11.90 